\title{
In-Orbit Performance of the GRACE Follow-on Laser Ranging Interferometer
}

Klaus Abich, ${ }^{1}$ Alexander Abramovici, ${ }^{2}$ Bengie Amparan, ${ }^{3}$ Andreas Baatzsch, ${ }^{4}$ Brian Bachman Okihiro, ${ }^{2}$ David C. Barr, ${ }^{2}$ Maxime P. Bize, ${ }^{2}$ Christina Bogan, ${ }^{5,}{ }^{*}$ Claus Braxmaier, ${ }^{1}$ Michael J. Burke, ${ }^{2}$ Ken C. Clark, ${ }^{2}$ Christian Dahl, ${ }^{4}$ Katrin Dahl, ${ }^{4}$ Karsten Danzmann, ${ }^{5}$ Mike A. Davis, ${ }^{3}$ Glenn de Vine, ${ }^{2}$ Jeffrey A. Dickson, ${ }^{2}$ Serge Dubovitsky, ${ }^{2}$ Andreas Eckardt, ${ }^{6}$ Thomas Ester, ${ }^{7}$ Germán Fernández Barranco, ${ }^{5}$ Reinhold Flatscher, ${ }^{8}$ Frank Flechtner, ${ }^{9,10}$ William M. Folkner, ${ }^{2}$ Samuel Francis, ${ }^{2}$ Martin S. Gilbert, ${ }^{2}$ Frank Gilles, ${ }^{4}$ Martin Gohlke, ${ }^{1}$ Nicolas Grossard, ${ }^{11}$ Burghardt Guenther, ${ }^{6}$ Philipp Hager, ${ }^{4, \dagger}$ Jerome Hauden, ${ }^{11}$ Frank Heine, ${ }^{7}$ Gerhard Heinzel, ${ }^{5, \$}$ Mark Herding, ${ }^{4}$ Martin Hinz, ${ }^{12}$ James Howell, ${ }^{3}$ Mark Katsumura, ${ }^{2}$ Marina Kaufer, ${ }^{4}$ William Klipstein, ${ }^{2}$ Alexander Koch, ${ }^{5}$ Micah Kruger, ${ }^{3}$ Kameron Larsen, ${ }^{2}$ Anton Lebeda, ${ }^{13}$ Arnold Lebeda, ${ }^{13}$ Thomas Leikert, ${ }^{12}$ Carl Christian Liebe, ${ }^{2}$ Jehhal Liu, ${ }^{2}$ Lynette Lobmeyer, ${ }^{3}$ Christoph Mahrdt, ${ }^{5,8}$ Thomas Mangoldt, ${ }^{6}$ Kirk McKenzie, ${ }^{2, \|}$ Malte Misfeldt, ${ }^{5}$ Phillip R. Morton, ${ }^{2}$ Vitali Müller, ${ }^{5}$ Alexander T. Murray, ${ }^{2}$ Don J. Nguyen, ${ }^{2}$ Kolja Nicklaus, ${ }^{4}$ Robert Pierce, ${ }^{3}$ Joshua A. Ravich, ${ }^{2}$ Gretchen Reavis, ${ }^{3}$ Jens Reiche, ${ }^{5}$ Josep Sanjuan, ${ }^{1}$ Daniel Schütze, ${ }^{5,9}$ Christoph Seiter, ${ }^{7}$ Daniel Shaddock, ${ }^{2, * *}$ Benjamin Sheard, ${ }^{5, \dagger}$

Michael Sileo, ${ }^{3}$ Robert Spero, ${ }^{2}$ Gary Spiers, ${ }^{2}$ Gunnar Stede, ${ }^{5, *}$ Michelle Stephens, ${ }^{3, \S}$ Andrew Sutton, ${ }^{2}$ Joseph Trinh, ${ }^{2}$ Kai Voss, ${ }^{4}$ Duo Wang, ${ }^{2}$ Rabi T. Wang, ${ }^{2}$ Brent Ware, ${ }^{2}$ Henry Wegener, ${ }^{5}$ Steve Windisch, ${ }^{7}$ Christopher Woodruff, ${ }^{2}$

Bernd Zender, ${ }^{6}$ and Marcus Zimmermann ${ }^{12}$

${ }^{1}$ Deutsches Zentrum für Luft- und Raumfahrt Institut für Raumfahrtsysteme, Robert-Hooke-Str. 7, 28359 Bremen, Germany

${ }^{2}$ Jet Propulsion Laboratory, California Institute of Technology, 4800 Oak Grove Drive, Pasadena, California 91109, USA

${ }^{3}$ Ball Aerospace and Technologies Corporation, PO Box 1062, Boulder, Colorado 80306, USA

${ }^{4}$ SpaceTech GmbH, Seelbachstrasse 13, 88090 Immenstaad, Germany

${ }^{5}$ Max-Planck-Institut für Gravitationsphysik (Albert-Einstein-Institut) and Institut für Gravitationsphysik of Leibniz Universität Hannover, Callinstrasse 38, 30167 Hannover, Germany

${ }^{6}$ Deutsches Zentrum für Luft- und Raumfahrt Institut für Optische Sensorsysteme, Rutherfordstrasse 2, 12489 Berlin-Adlershof, Germany

${ }^{7}$ Tesat-Spacecom GmbH \& Co KG, Gerberstr. 49, 71522 Backnang, Germany

${ }^{8}$ Airbus Defence \& Space, 88039 Friedrichshafen, Germany

${ }^{9}$ Deutsches GeoForschungsZentrum GFZ, Telegrafenberg, 14473 Potsdam, Germany

${ }^{10}$ Technische Universität Berlin, Strasse des 17. Juni 135, 10623 Berlin, Germany

${ }^{11}$ iXblue Photonics, 34 rue de la Croix de fer, 78100 Saint Germain en Laye, France

${ }^{12}$ Hensoldt Optronics GmbH, Carl-Zeiss-Straße 22, 73447 Oberkochen, Germany

${ }^{13}$ APCON AeroSpace \& Defence, Prof. Messerschmitt-Str. 10, 85579 Neubiberg, Germany

(Received 15 April 2019; revised manuscript received 1 June 2019; published 19 July 2019)

The Laser Ranging Interferometer (LRI) instrument on the Gravity Recovery and Climate Experiment (GRACE) Follow-On mission has provided the first laser interferometric range measurements between remote spacecraft, separated by approximately $220 \mathrm{~km}$. Autonomous controls that lock the laser frequency to a cavity reference and establish the 5 degrees of freedom two-way laser link between remote spacecraft succeeded on the first attempt. Active beam pointing based on differential wave front sensing compensates spacecraft attitude fluctuations. The LRI has operated continuously without breaks in phase tracking for more than 50 days, and has shown biased range measurements similar to the primary ranging instrument based on microwaves, but with much less noise at a level of $1 \mathrm{~nm} / \sqrt{\mathrm{Hz}}$ at Fourier frequencies above $100 \mathrm{mHz}$.

DOI: 10.1103/PhysRevLett.123.031101

Introduction.-The Gravity Recovery and Climate Experiment (GRACE) mission [1,2], in orbit from 2002-2017, was a U.S.-German collaboration that has

Published by the American Physical Society under the terms of the Creative Commons Attribution 4.0 International license. Further distribution of this work must maintain attribution to the author(s) and the published article's title, journal citation, and DOI. revolutionized the measurement of the time-variable Earth gravity field. The primary instrument was the microwave instrument (MWI) that tracked the variations of the distance (biased range) between the two satellites as they followed each other with $200 \mathrm{~km}$ separation on a near polar orbit about $450 \mathrm{~km}$ above Earth. These variations reflect the structure of the gravity field and additionally contain small nongravitational accelerations.

Data processing of the range variations that also involved the on-board accelerometer, GPS-based precise 
orbit determination, modeling of ocean and solid Earth tides, and other smaller effects yielded as the main result monthly maps of Earth's gravity field. GRACE data have been used by hundreds of researchers worldwide in the publication of thousands of papers in climate research, tracking changes in ice and ground water, in geophysics, and in many other fields of research. Desire to continue the 15 years of observations led to GRACE Follow-On-which is again a U.S.-German partnership and in key aspects a rebuild of GRACE with again the MWI as the main instrument [3,4]. The main new feature of GRACE Follow-On is the addition of the laser ranging interferometer (LRI) instrument as a technology demonstrator for future Earth-science missions. The LRI also serves as a useful demonstrator for the Laser Interferometer Space Antenna (LISA) [5]. It measures the same variations in the inter-spacecraft distance as the MWI, but with less noise [6,7].

These variations have amplitudes of a few hundred meters at the orbital frequency. They contain nongravitational disturbances and the gravity signal that spans a huge dynamic range between $10^{-8}$ and $1 \mathrm{~m} / \sqrt{\mathrm{Hz}}$ (Figs. 4 and 6). Most of that signal encodes the static Earth's gravity field. The temporal variations are much smaller [8], motivating the need for ranging noise in the $\mathrm{nm} / \sqrt{\mathrm{Hz}}$ range.

The LRI is the first laser interferometer to be operated between distant satellites. GRACE Follow-On was launched on May 22, 2018 and the first attempt to turn on the LRI took place on June 14, with immediate success. This Letter summarizes the design of the LRI, discusses the acquisition strategy, and reports the first ranging measurements.

LRI components and design.-Figure 1 shows a functional diagram of the LRI on the two identical spacecraft. Its components include the laser, the cavity, the laser ranging processor (LRP), the optical bench electronics (OBE), the optical bench assembly (OBA), and the triple mirror assembly (TMA). The basic design of the LRI has been described in a separate paper [6].

The LRI is a U.S.-German cooperative project led by NASA/JPL in the U.S. and the AEI in Hannover, Germany. The laser, cavity, and LRP were contributed by the U.S., while the TMA, OBA, and OBE were contributed by Germany.

The lasers are Nd:YAG nonplanar ring oscillators manufactured by Tesat-Spacecom. They operate at a wavelength of $1064.5 \mathrm{~nm}$ and produce $25 \mathrm{~mW}$ of fiber coupled laser light. The frequency stability of the laser limits the sensitivity of the LRI instrument at high frequencies. Therefore, both spacecraft carry identical optical cavities, one of which is used to stabilize the laser frequency on the spacecraft designated as master. The flight units of the optical cavities were manufactured by Ball Aerospace based on prototypes developed by Ball Aerospace and JPL under a NASA Earth Science grant $[9,10]$. Each cavity unit includes an optical phase modulator delivered by iXblue. Ground measurements of the respective LRI components showed frequency noise below $30 \mathrm{~Hz} / \sqrt{\mathrm{Hz}}$ at Fourier frequencies of $1 \mathrm{mHz}$ and above [11].

The LRP was developed and built by JPL, based on LISA and Earth Science studies [9-12]. It processes the photoreceiver signals both by tracking with digital phaselocked loops for the ranging measurements (phasemeter function) and by continuously running a fast Fourier transform (FFT), which is used in particular during acquisition. The LRP commands the fast steering mirror in link acquisition and performs closed loop control for differential wave front sensing when the interspacecraft

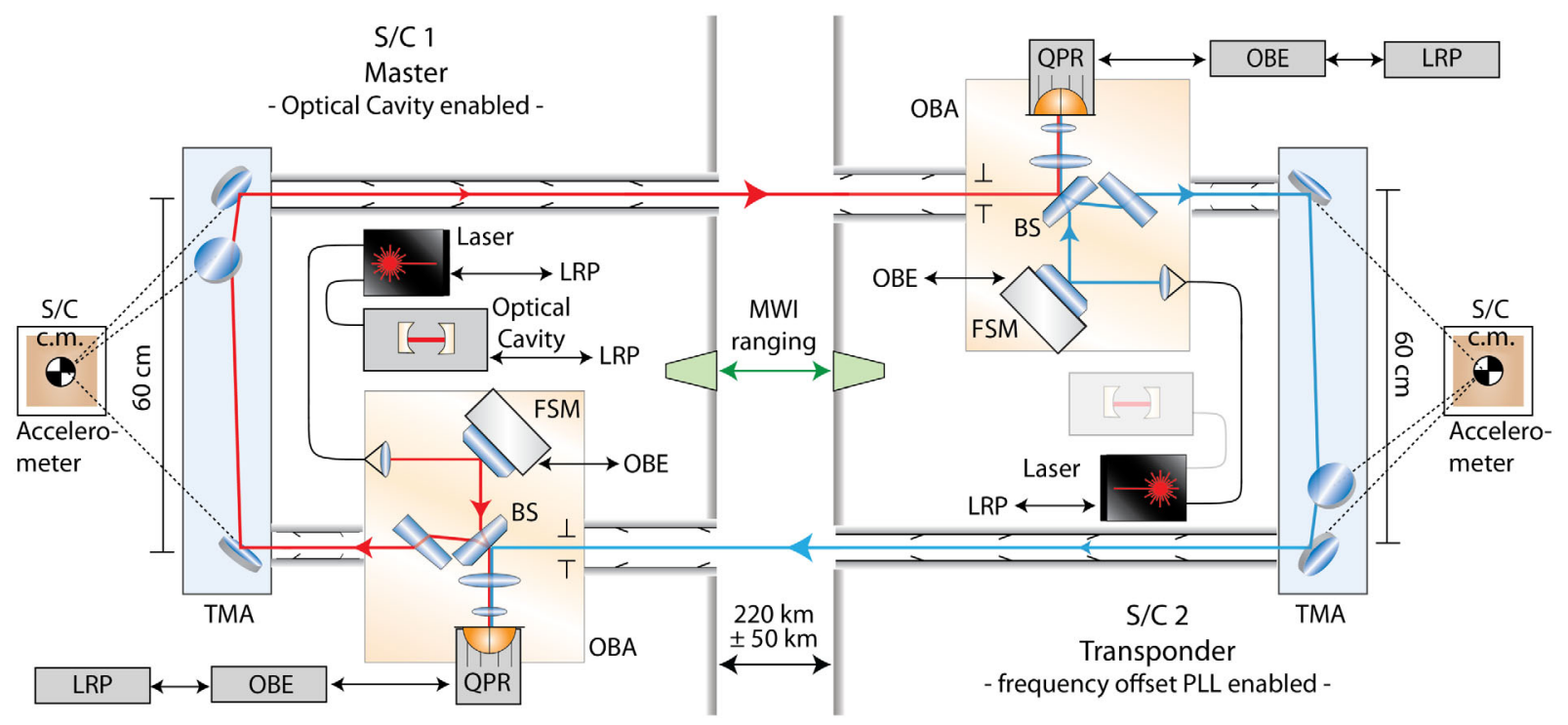

FIG. 1. Functional overview of the LRI units on both spacecraft. The LRI units include the laser, cavity, laser ranging processor (LRP), optical bench electronics (OBE), triple mirror assembly (TMA), and optical bench assembly (OBA) with a fast steering mirror (FSM). 
link is active. It controls the laser frequency as either master or transponder. The LRI's command and telemetry is handled by the LRP.

The LRI optical system consists of the OBA [13] built by SpaceTech GmbH (STI) with associated OBE built by the Deutsches Zentrum für Luft- und Raumfahrt (DLR) in Adlershof, the TMA from STI and Hensoldt/Zeiss, and associated baffles and mechanical parts (from STI).

On the optical bench, which is relatively simple compared to LISA Pathfinder [14,15] or LISA, light from the laser is delivered through an optical single-mode fiber and custom-made collimator to produce a Gaussian beam of $2.5 \mathrm{~mm}$ radius. It is routed via a fast steering mirror (FSM) [16] from Airbus Defence and Space which can steer the beam direction by several mrad in two axes at rates $>100 \mathrm{~Hz}$. A beam splitter (BS) transmits $10 \%$ of the light to be used as the local oscillator (LO) to enable heterodyne detection of the $\mathrm{nW}$-level received (RX) light. The latter passes through an aperture of $8 \mathrm{~mm}$ diameter before arriving at the other port of the BS. The aperture and steering mirror have the same distance from the BS. A twolens imaging system images both with a magnification $m=1 / 8$ on to the InGaAs quadrant photoreceiver (QPR) such that a tilt of either FSM or the incoming wave front translates into an $8 \times$ magnified tilt on the QPR with virtually no beam walk. The remaining $90 \%$ of the local laser light is reflected by the $\mathrm{BS}$ and is routed through the TMA, which consists of three plane mirrors, whose planes are precisely orthogonal to each other and intersect in a virtual vertex located at the center of mass (c.m.) of the accelerometer test mass, the reference point for the ranging measurement [17-20]. The TMA reverses the direction of the beam and directs it towards the other spacecraft and also displaces it by $60 \mathrm{~cm}$ to avoid the vicinity of the central line of sight, which is occupied by other equipment. The divergence of the transmitted (TX) light of $140 \mu \mathrm{rad}$ causes the beam to expand to about $30 \mathrm{~m}$ radius, from which a tiny fraction (order of $\mathrm{nW}$ ) is cut out with the $\mathrm{RX}$ aperture at the other end. One spacecraft acts as master, sending out a laser beam with stabilized frequency. The other spacecraft acts as a transponder and sends back a laser beam that is phase locked with a fixed $10 \mathrm{MHz}$ offset to the weak incoming light. Both spacecraft are identical and can be commanded to either the master or transponder role. The total mass of the LRI units per spacecraft is $25 \mathrm{~kg}$, and the nominal power consumption is $35 \mathrm{~W}$.

The incoming light interferes with the LO, resulting in a beat note around $10 \mathrm{MHz}$, the phase of which is recorded with the phasemeter function of the LRP. The orbital motion contains relative velocities along the line of sight with an amplitude of $\pm 0.5 \mathrm{~m} / \mathrm{s}$, giving rise to Doppler shifts of $\pm 0.5 \mathrm{MHz}$, which also contain the gravity signal of interest [21]. On the transponder spacecraft, the offsetphase lock impresses that Doppler shift on the retransmitted light, which picks up one more Doppler shift on its way

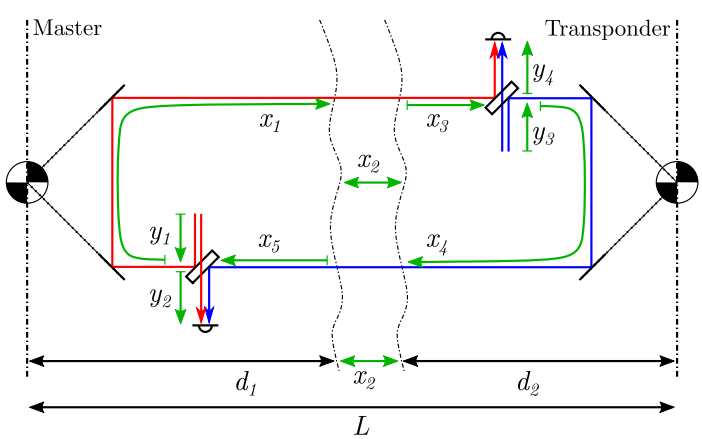

FIG. 2. LRI measured lengths.

back. As a result, the phasemeter on the transponder records a constant $10 \mathrm{MHz}$ tone, while the beat note on the master additionally contains twice the Doppler shift.

This is different from the dual one-way ranging system employed by the MWI, but similar to the laser interferometry envisaged for LISA [22]. An analysis of the optical path lengths shown in Fig. 2 reveals that the effectively measured quantity is $x_{1}+x_{2}+x_{3}+x_{4}+x_{2}+x_{5}=$ $2\left(d_{1}+d_{2}+x_{2}\right)=2 L$, i.e., twice the separation $L$ of the two TMA vertices, while all other lengths $\left(y_{1} \ldots y_{4}\right.$ as well as the delivery fibers, etc.) cancel to first order [6].

A specific feature of the LRI is its beam pointing function [23], which is required because of the narrower beam width of $140 \mu \mathrm{rad}$ half-cone angle compared to the microwave beam of $30 \mathrm{mrad}$.

The LRI transmit beam must point to the other distant spacecraft with better than $100 \mu \mathrm{rad}$ accuracy to ensure that enough light arrives at the distant RX aperture. Similarly, the weak RX beam must be aligned to the LO beam to about the same accuracy in order to achieve sufficient heterodyne efficiency. The attitude fluctuations of the spacecraft with respect to the line of sight are up to 3 times larger than that, thus requiring active compensation. Accurate beam pointing is achieved by a single actuator, the FSM, together with differential wave front sensing [24] signals and the retroreflector properties of the TMA $[17,23]$.

The requirement for the LRI ranging noise was set to $80 \mathrm{~nm} / \sqrt{\mathrm{Hz}}$ between 2 and $100 \mathrm{mHz}$, with a noise-shape function of (Fig. 5)

$$
\tilde{s}<80 \mathrm{~nm} / \sqrt{\mathrm{Hz}} \sqrt{1+\left(\frac{3 \mathrm{mHz}}{f}\right)^{2}} \sqrt{1+\left(\frac{10 \mathrm{mHz}}{f}\right)^{2}} .
$$

This is 1 order of magnitude better than the instrumental noise of the MWI, and was designed to be low enough such that it is not the limiting noise source at any frequency. The gravity field resolution is expected to be limited by other noise sources such as tide modeling and aliasing [7].

The LRI sensitivity was designed to be limited at higher frequencies by laser frequency noise scaled with the $\approx 220 \mathrm{~km}$ separation, and at lower frequencies by 


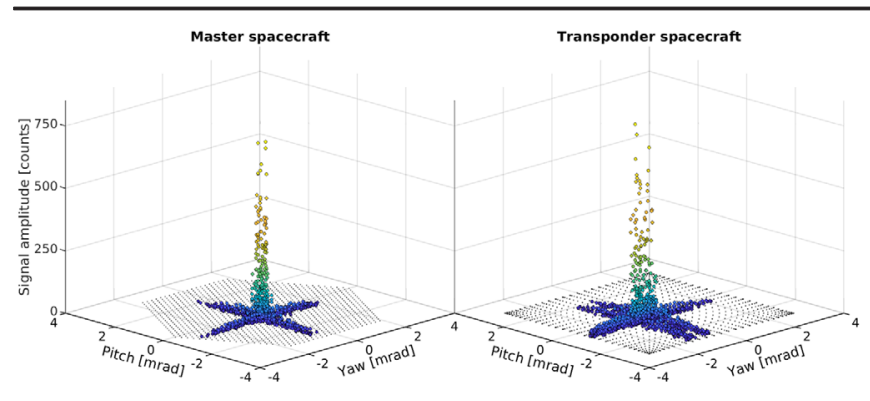

FIG. 3. FFT amplitude peaks from the initial acquisition scan on June 13, 2018. Nonzero values represent the instances $(<1 \mathrm{sec})$ during the $8.5 \mathrm{~h}$ scan when all 5 degrees of freedom were near their correct values and therefore a heterodyne signal was detected.

tilt-to-length coupling originating from the spacecraft attitude jitter in combination with coupling factors of a few $100 \mu \mathrm{m} / \mathrm{rad}$ originating from alignment tolerances.

The LRI instrument operates autonomously and produces continuous range telemetry at a $\approx 10$ samples per second update rate to be used for gravity field recovery.

The similarities to the planned LISA mission include the measurement band $(\mathrm{mHz})$, the heterodyne frequency $(\mathrm{MHz})$, the received light power level $(\mathrm{nW})$, the offset locked transponder scheme, the digital phase-locked loop phasemeter, the laser frequency stability achieved by a stable external cavity, autonomous locking procedures for the cavity and the transponder loop, the quadrant photoreceivers with differential wave front sensing, and the principle of the 5 degrees of freedom acquisition procedure. In spite of remaining differences, this first successful demonstration of long distance spacecraft interferometry is thus highly relevant for the ongoing design of LISA as well.

Link acquisition.-Early in the development of the LRI, link acquisition was identified as a critical function and it has therefore been intensively studied [25-28]. Link acquisition requires that 5 degrees of freedom are simultaneously within narrow ranges around their optimum. These are two pointing angles (pitch and yaw) per spacecraft, each of which has to be within $\pm 100 \mu \mathrm{rad}$, and the difference between the two absolute laser frequencies which has to fulfill $|\Delta f|<15 \mathrm{MHz}$ due to the photoreceiver and phasemeter bandwidths. The unknown offsets in these quantities are approximately 10 times larger after launch. In the initial acquisition scan, an exhaustive scan of the 5D parameter space is performed over the course of several hours. Its purpose is to determine the static pointing offsets between the LRI optical axes and the nominal spacecraft pointing direction, and the frequency difference between the two lasers. The LRPs on both spacecraft continuously compute FFT spectra of the QPR samples $(N=4096$ at $f_{\text {samp }} \approx 39 \mathrm{MHz}$ ), resulting in power measurements roughly every $100 \mu$ s for each frequency bin of $10 \mathrm{kHz}$ width. Peaks in the spectra are identified and stored. These are downloaded along with the corresponding commanded

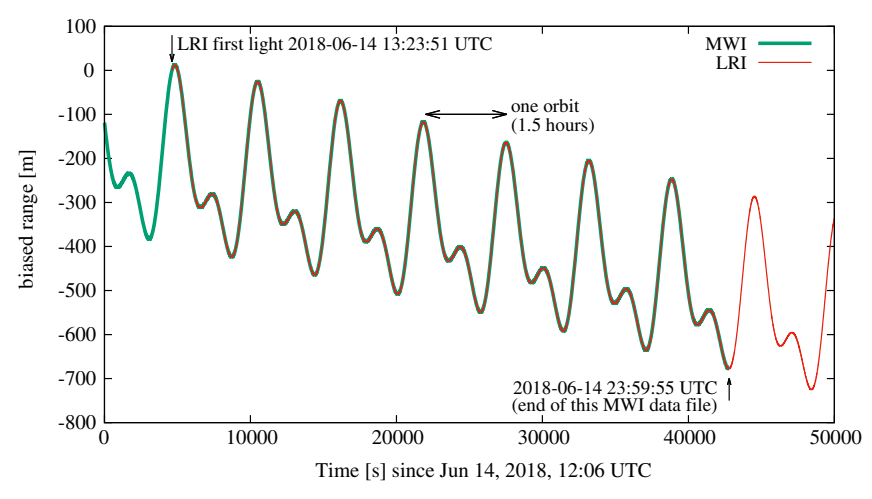

FIG. 4. First LRI ranging measurements show good agreement with the MWI.

positions of the FSM and the laser frequency set point and analyzed on ground (Figure 3).

The scan patterns are a slow hexagonal pattern on the master spacecraft and a fast Lissajous pattern on the transponder spacecraft. They are added to the reference direction that is provided by the spacecraft as an estimate of its attitude error. The slowest outermost actuation scans the transponder laser frequency, while the master stays locked on a cavity resonance. The angular scan range is approx. $\pm 3 \mathrm{mrad}$. The fast Lissajous pattern is scanned at 100 and $2 \mathrm{~Hz}$ in the two axes, respectively, while the hexagonal pattern consists of 1200 discrete points with a hold time of $0.56 \mathrm{~s}$ per point, resulting in a duration of 11.7 min for a single hexagonal master scan during the initial acquisition phase. The transponder laser frequency sweeps the $320 \mathrm{MHz}$ peak-peak uncertainty range with $10.5 \mathrm{kHz} / \mathrm{s}$, which yields a duration of approx. $8.5 \mathrm{~h}$ for the full initial acquisition scan.

The downlinked data are processed to determine the angular and frequency offsets. These offsets are uploaded and then the instrument is commanded into reacquisition mode, which performs a similar $5 \mathrm{D}$ scan, but with a 10 times reduced range $( \pm 300 \mu \mathrm{rad})$. When the LRI detects a heterodyne signal it stops the scanning and transitions to the phase tracking mode. Reacquisition takes less than 5 min.

On-orbit performance.-GRACE Follow-On was launched on May 22, 2018 by a SpaceX Falcon-9 launch vehicle from the Vandenberg Air Force Base, California, together with five Iridium-Next satellites. Contact was established immediately after separation from the second stage, $11 \mathrm{~min}$ after launch. Orbit insertion was nearly perfect. After routine checkout procedures of the spacecraft, the MWI system, and the individual LRI components, controlled from the German Space Operations Center (GSOC) in Oberpfaffenhofen, the initial acquisition scan was started on June 13, 2018. Many peaks of high signalto-noise ratio were found (Fig. 3). The slow scan on the master yielded a single maximum, while the fast Lissajous scan on the transponder produced several maxima due to 


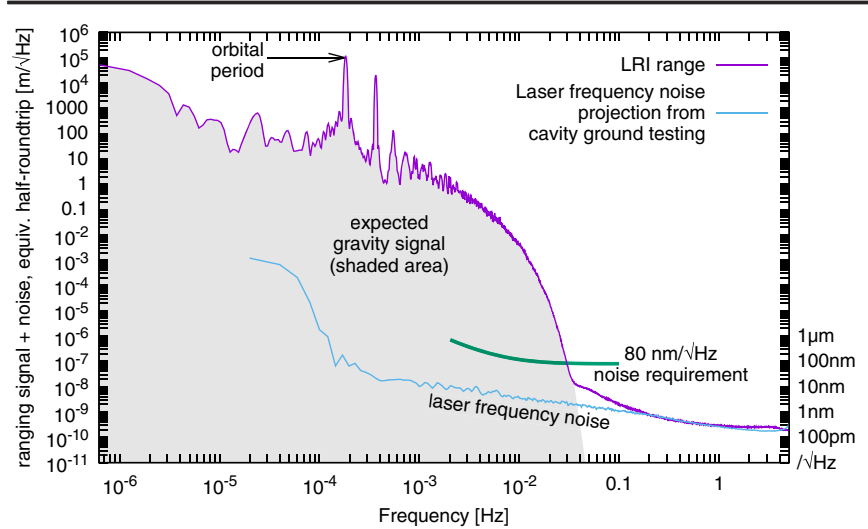

FIG. 5. Amplitude spectral density of LRI ranging measurements. The purple line shows the ranging signal, after subtraction of phase jumps, which is dominated by the gravity signal below $30 \mathrm{mHz}$. The blue line shows the stabilized laser frequency noise projected from ground measurements and the green line shows the LRI requirement.

the delay between the FSM-commanded position and FFT peak finding, which was expected and corrected in the immediately performed data analysis on the ground. Equally expected were the "+" shaped sidelobes, which stem from the slits in the QPRs [25]. The resulting pointing offsets as determined by the maxima in Fig. 3 were on the order of $0.5-1 \mathrm{mrad}$. The calculated position and frequency offsets were uplinked, along with the command to enter reacquisition mode, on June 14. On the next downlink, both spacecraft reported that they had entered science mode on the first attempt. The LRI stayed in science data collection mode with no interruptions of the links other than intentional mode switches or spacecraft activities unrelated to the LRI. The few instances of reacquisition were all immediately successful within a few minutes. The longest segment without any interruption of the link during the first months of operation was 55.5 day, about 850 orbits. The immediate comparison of the LRI range and MWI range is shown in Fig. 4 and confirms that the LRI measures the biased inter-spacecraft range. While the LRI was designed and verified on ground to operate with a carrier-to-noise ratio (CNR) of as low as $70 \mathrm{~dB}-\mathrm{Hz}$, the observed CNR during initial operation was $88 \mathrm{~dB}-\mathrm{Hz}$, providing ample margin.

Figure 5 shows the amplitude spectral density of the LRI range measurement, where the only processing was the removal of phase jumps (see below).

It contains the full gravity signal and all nongravitational observations. At high frequencies, it is limited by laser frequency noise, in good agreement with ground measurements of the cavity performance, which translates to, e.g., $\approx 4 \mathrm{~nm} / \sqrt{\mathrm{Hz}}$ at $0.1 \mathrm{~Hz}$. Towards higher frequencies, the noise level falls off to values as low as $0.2 \mathrm{~nm} / \sqrt{\mathrm{Hz}}$.

The measured phase has occasional jumps coincident with attitude control thruster activation, which occur
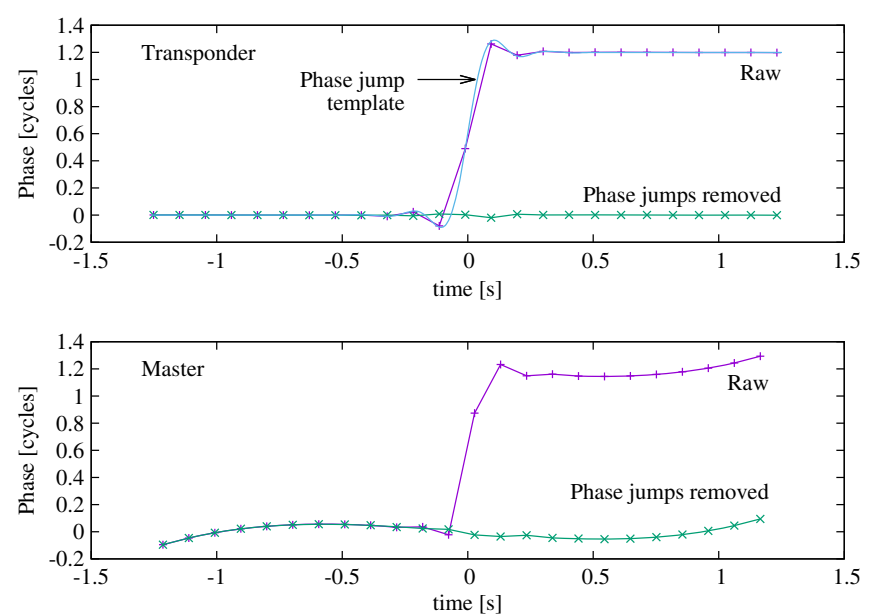

FIG. 6. The LRI instrument exhibits occasional phase jumps in the inter-spacecraft range measurement. These are correlated with thruster activations. The analysis shows that phase tracking is always maintained and the phase jumps can always be removed without any impact on the range data. A polynomial of degree 1 and 2 was subtracted from transponder and master phase, respectively, to make the jumps visible in the plot.

typically a few times per orbit revolution. These are modeled by a step convolved with the final antialiasing filter response, and removed from the data, with residuals below the noise floor (Fig. 6). Thus, they have no effect upon the performance of the instrument.

Further analysis of the LRI ranging performance below $0.1 \mathrm{~Hz}$ will be possible from the residuals after the gravity field recovery, which is ongoing work.

Conclusion.-Since initial turn on, the LRI has continuously returned range data, interrupted only by spacecraft operation. Its noise at frequencies that can be evaluated directly is well below the requirement, reaching $10 \mathrm{~nm} / \sqrt{\mathrm{Hz}}$ at $40 \mathrm{mHz}$ and $300 \mathrm{pm} / \sqrt{\mathrm{Hz}}$ at $1 \mathrm{~Hz}$. The inband noise level will only be accessible after full gravity field analysis. In addition to providing a low-noise laser ranging measurement demonstration for future geodesy missions, the LRI also demonstrates inter-spacecraft interferometry for LISA.

The LRI team would like to acknowledge the GRACE Follow-On Project teams at JPL including project management, mission assurance and quality assurance, project and flight system engineering, mission operations and science data systems at JPL and CSR. It is a pleasure to acknowledge the spacecraft provider, Airbus Defence and Space, for their excellence in integrating the LRI instrument to precise requirements without which the LRI could not meet its goals. The LRI team would like to acknowledge the Deutsches Zentrum für Luft und Raumfahrt (DLR) and German Research Centre for Geosciences (GFZ) Mission Operation teams at the German Space Operation Center (GSOC) for their excellent work and support in 
commanding the LRI instrument in all mission phases. The Australian National University developed an alternative triple mirror assembly and were involved in testing and concept development for link acquisition. Part of the research was carried out at the Jet Propulsion Laboratory, California Institute of Technology, under a contract with the National Aeronautics and Space Administration. GRACE-FO is a partnership between NASA and German Research Centre for Geosciences in Potsdam, Germany. JPL manages the mission for NASA's Science Mission Directorate. The development of the LRI in Germany was supported by Bundesministerium für Bildung und Forschung (BMBF), Project No. 03F0654B, Deutsche Forschungsgemeinschaft (DFG) and Deutsches Zentrum für Luft- und Raumfahrt (DLR).

\footnotetext{
*Present address: Volkswagen AG, Wolfsburg, Germany. ${ }^{\dagger}$ Present address: ESA ESTEC, Noordwijk, Netherlands. * Corresponding author. gerhard.heinzel@aei.mpg.de

${ }^{\S}$ Present address: Siemens Mobility GmbH, Braunschweig, Germany.

Corresponding author.

kirk.mckenzie@jpl.nasa.gov

"Present address: OHB-Systems AG, Bremen, Germany. ** Present address: Liquid Instruments, Canberra Australia.

"†Present address: OHB-Systems AG, Bremen, Germany.

\# Present address: Leibnizschule, Hannover, Germany.

${ }^{\S}$ Present address: NIST, Boulder, USA.
}

[1] B. D. Tapley, S. Bettadpur, J. C. Ries, P. F. Thompson, and M. M. Watkins, Grace measurements of mass variability in the earth system, Science 305, 503 (2004).

[2] B. Tapley, J. Ries, S. Bettadpur, D. Chambers, M. Cheng, F. Condi, B. Gunter, Z. Kang, P. Nagel, R. Pastor, T. Pekker, S. Poole, and $\mathrm{F}$. Wang, $\mathrm{GGMO}_{2}-\mathrm{An}$ improved earth gravity field model from GRACE, J. Geodes. 79, 467 (2005).

[3] F. Flechtner, P. Morton, M. Watkins, and F. Webb, Status of the GRACE follow-on mission, in Gravity, Geoid and Height Systems, International Association of Geodesy Symposia Vol. 141, edited by U. Marti (Springer, Cham, 2014), pp. 117-121.

[4] S. Kayali, P. Morton, and M. Gross (IEEE), International challenges of GRACE follow-on, in 2017 IEEE Aerospace Conference, IEEE Aerospace Conference Proceedings (IEEE, New York, 2017), pp. 1-8, https://ieeexplore.ieee .org/document/7943615.

[5] European Space Agency, Laser Interferometer Space Antenna, http://sci.esa.int/lisa/ (2019).

[6] B. S. Sheard, G. Heinzel, K. Danzmann, D. A. Shaddock, W. M. Klipstein, and W. M. Folkner, Intersatellite laser ranging instrument for the GRACE follow-on mission, J. Geodes. 86, 1083 (2012).

[7] F. Flechtner, K.-H. Neumayer, C. Dahle, H. Dobslaw, E. Fagiolini, J.-C. Raimondo, and A. Guentner, What can be expected from the GRACE-FO laser ranging interferometer for earth science applications? Surv. Geophys. 37, 453 (2016).

[8] T. Gruber, J. L. Bamber, M. F. P. Bierkens, H. Dobslaw, M. Murböck, M. Thomas, L. P. H. van Beek, T. van Dam, L. L. A. Vermeersen, and P. N. A. M. Visser, Simulation of the time-variable gravity field by means of coupled geophysical models, Earth Syst. Sci. Data 3, 19 (2011).

[9] R. Pierce, M. Stephens, P. Kaptchen, J. Leitch, D. Bender, W. M. Folkner, W. M. Klipstein, D. Shaddock, R. Spero, R. Thompson, N. Yu, and M. Watkins (IEEE), Stabilized lasers for space applications: A high TRL optical cavity reference system, in 2012 Conference on Lasers and Electro-Optics, Conference on Lasers and Electro-Optics (IEEE, New York, 2012), pp. 1-2, https://doi.org/10.1364/CLEO_AT.2012 .JW3C.3.

[10] R. Thompson, W. M. Folkner, G. deVine, W. M. Klipstein, K. McKenzie, R. Spero, N. Yu, M. Stephens, J. Leitch, R. Pierce, T. T. Y. Lam, andD. A. Shaddock (IEEE), A flightlike optical reference cavity for GRACE Follow-On laser frequency stabilization, in 2011 Joint Conference of the IEEE International Frequency Control Symposium/ European Frequency and Time Forum Proceedings, IEEE International Frequency Control Symposium (IEEE, New York, 2011), pp. 729-731, https://ieeexplore.ieee.org/ document/5977873.

[11] B. Bachman, G. de Vine, J. Dickson, S. Dubovitsky, J. Liu, W. Klipstein, K. McKenzie, R. Spero, A. Sutton, B. Ware, and C. Woodruff (IOP), Flight phasemeter on the laser ranging interferometer on the GRACE Follow-On mission, in Proceedings of the 11th International LISA Symposium, Journal of Physics Conference Series Vol. 840 (IOP Publishing, Bristol, 2017), p. 012011, https://doi.org/10.1088/ 1742-6596/840/1/012011.

[12] D. Shaddock, B. Ware, P. G. Halverson, R. E. Spero, and B. Klipstein, Overview of the LISA phasemeter, AIP Conf. Ser. 873, 654 (2006).

[13] K. Nicklaus, M. Herding, A. Baatzsch, M. Dehne, C. Diekmann, K. Voss, F. Gilles, B. Guenther, B. Zender, S. Boehme et al., Optical bench of the laser ranging interferometer on GRACE Follow-On, in International Conference on Space OpticsICSO 2014 (SPIE, Bellingham, 2017), Vol. 10563, p. 105632I, https://doi.org/10.1117/12.2304195.

[14] M. Armano et al., Beyond the Required LISA Free-Fall Performance: New LISA Pathfinder Results Down to $20 \mu \mathrm{Hz}$, Phys. Rev. Lett. 120, 061101 (2018).

[15] C. J. Killow, E. D. Fitzsimons, J. Hough, M. Perreur-Lloyd, D. I. Robertson, S. Rowan, and H. Ward, Construction of rugged, ultrastable optical assemblies with optical component alignment at the few microradian level, Appl. Opt. 52, 177 (2013).

[16] H. Langenbach and M. Schmid, Fast steering mirror for laser communication, in Proceedings of the 11th European Space Mechanisms and Tribology Symposium, ESA Special Publications Vol. 591, edited by B. Warmbein (ESA Publications Division, Noordwijk, Netherlands, 2005), pp. 27-33.

[17] R. L. Ward et al., The design and construction of a prototype lateral-transfer retro-reflector for inter-satellite laser ranging, Classical Quantum Gravity 31, 095015 (2014).

[18] D. Schuetze, V. Mueller, G. Stede, B. S. Sheard, G. Heinzel, K. Danzmann, A. J. Sutton, and D. A. Shaddock, Retroreflector for GRACE follow-on: Vertex vs. point of minimal coupling, Opt. Express 22, 9324 (2014).

[19] D. Schuetze, D. Farrant, D. A. Shaddock, B. S. Sheard, G. Heinzel, and K. Danzmann, Measuring coalignment of 
retroreflectors with large lateral incoming-outgoing beam offset, Rev. Sci. Instrum. 85, 035103 (2014).

[20] D. Schuetze, G. Stede, V. Mueller, O. Gerberding, C. Mahrdt, B. Sheard, G. Heinzel, and K. Danzmann, in LISA-like Laser Ranging for GRACE Follow-On, 9th Lisa Symposium Vol. 467 (Astronomical Society of the Pacific, San Francisco, 2013), pp. 285-290.

[21] G. Seeber, Satellite Geodesy (Walter de Gruyter, Berlin, 2003).

[22] J. I. Thorpe, LISA long-arm interferometry, Classical Quantum Gravity 27, 084008 (2010).

[23] D. Schuetze, G. Stede, V. Mueller, O. Gerberding, T. Bandikova, B.S. Sheard, G. Heinzel, and K. Danzmann, Laser beam steering for GRACE Follow-On intersatellite interferometry, Opt. Express 22, 24117 (2014).

[24] E. Morrison, B. J. Meers, D. I. Robertson, and H. Ward, Automatic alignment of optical interferometers, Appl. Opt. 33, 5041 (1994).
[25] C. Mahrdt, Laser link acquisition for the GRACE follow-on laser ranging interferometer, Ph.D. thesis, Gottfried Wilhelm Leibniz Universität Hannover, 2014.

[26] D. M. R. Wuchenich, C. Mahrdt, B. S. Sheard, S. P. Francis, R. E. Spero, J. Miller, C. M. Mow-Lowry, R. L. Ward, W. M. Klipstein, G. Heinzel, K. Danzmann, D. E. McClelland, and D. A. Shaddock, Laser link acquisition demonstration for the GRACE Follow-On mission, Opt. Express 22, 11351 (2014).

[27] J. Sanjuan, M. Gohlke, S. Rasch, K. Abich, A. Goerth, G. Heinzel, and C. Braxmaier, Interspacecraft link simulator for the laser ranging interferometer onboard GRACE Follow-On, Appl. Opt. 54, 6682 (2015).

[28] A. Koch, J. Sanjuan, M. Gohlke, C. Mahrdt, N. Brause, C. Braxmaier, and G. Heinzel, Line of sight calibration for the laser ranging interferometer on-board the GRACE Follow-On mission: on-ground experimental validation, Opt. Express 26, 25892 (2018). 